\title{
Reparación de una perforación valvular mitral como secuela de endocarditis, aporte de la ecocardiografía tridimensional
}

\author{
Julián Vega, Samuel Córdova, María Cecilia Muñoz, Luigi Gabrielli, Paul McNab, Rodrigo Saavedra. \\ División de Enfermedades Cardiovasculares. Pontificia Universidad Católica de Chile. \\ *Sin apoyo financiero
}

Resumen Reportamos un caso de reparación de perforación valvular mitral en un joven de 18 años que consulta por disnea de esfuerzos y con el antecedente remoto de una artritis séptica de rodilla tratada. En el examen físico se encontró un soplo holosistólico $4 / 6$ en el foco mitral. Se realizó un ecocardiograma transtorácico (ETT) que objetivó una insuficiencia mitral severa, con un jet de recorrido muy excéntrico originado desde anterior, sospechando una perforación del velo anterior, sin lograr caracterizarla por dicha técnica. Se complementó el estudio con un ecocardiograma transesofágico
(ETE) en una plataforma EPIQ $7 \mathrm{cv}$ (PHILIPS), que identificó en el cuerpo del segmento 2 del velo anterior mitral (A2) una posible perforación. El análisis de la válvula mediante ETE tridimensional (3D), confirmó una perforación circular de bordes netos, de dimensiones máximas $6 \times 6 \mathrm{~mm}$, localizada en el cuerpo de A2. El análisis 3D aportó valiosa información para programar la reparación valvular, la cual se efectuó mediante un parche de pericardio autólogo fresco (sin fijación en glutaraldehido) y una anuloplastía con un anillo rígido, con óptimo resultado y sin complicaciones. 


\section{Mitral leaflet perforation repair after healed endocarditis, advantage of three-diminesional echocardiography}

An 18 year-old man presented with shortness of breath and a remote history of a septic arthritis of the knee. Physical examination revealed a holosystolic mitral valve murmur. Transthoracic echocardiography showed a severe mitral regurgitation originating from de anterior mitral valve leaflet, but the exact mechanism was unclear. Transesophageal echocardiography (EPIQ 7v, Philips) suggested a perforation of the medial scallop of the anterior mitral valve leaflet (A2). 3-D trans esophageal echocardiography confirmed a $6 \times 6 \mathrm{~mm}$ perforation at the A2 sector of the anterior leaflet. 3-D echo was also very helpful in guiding the surgical repair of this lesion, using a pericardial patch and rigid mitral valve ring.

Keywords: endocarditis; valvular mitral regurgitation; echocardiography, three dimensional; mitral valve repair. 
Caso: Reportamos un caso de reparación de una perforación mitral en un joven de 18 años que consulta por disnea de esfuerzos. Tenía el antecedente de haber sufrido a los 9 años de edad una artritis séptica de rodilla tratada con drenaje quirúrgico y terapia antibiótica intravenosa prolongada. Sin otros antecedentes mórbidos. Permanece asintomático y desarrollando actividad deportiva competitiva (básquetbol) con buena capacidad funcional hasta los 17 años de edad, cuando nota un progresivo deterioro en su rendimiento deportivo, caracterizado por disnea a moderados esfuerzos. Consulta pesquisándose al examen físico un soplo holosistólico de intensidad $4 / 6$ en el foco mitral, como único hallazgo.

Se realizó una ecocardiografía transtorácica (ETT) que informó una leve dilatación ventricular izquierda con buena motilidad global y segmentaria con aspecto de sobrecarga de volumen, junto a una insuficiencia mitral severa, con un jet de recorrido muy excéntrico originado desde anterior, sospechando una perforación del velo anterior, pero sin lograr caracterizar mayormente la lesión por dicha técnica. Posteriormente, se realizó una ecocardiografía transesofágica (ETE) en una plataforma EPIQ $7 \mathrm{cv}$ (Philips), que confirmó los hallazgos ya descritos en las cavidades cardíacas izquierdas. En cuanto a la válvula mitral, esta presentaba sus velos engrosados e hiperecogénicos; el velo posterior hacia su borde libre tenía un aspecto "deflecado" y en el velo anterior, específicamente en el cuerpo del segmento A2, se originaba un jet de insuficiencia de severa cuantía. En el barrido de la válvula por ETE bidimensional (2D) se sospechó una perforación a dicho nivel, sin lograr determinar claramente su tamaño y ubicación (Figura 1). No se encontraron otras lesiones valvulares, ni murales.

Se realizó el análisis de la válvula mediante ETE tridimensional (3D). Para ello se adquirió la imagen de la válvula mitral desde un eje largo medio-esofágico a partir de un "zoom 3D" optimizado para obtener el máximo de volúmenes por segundo (>12 Hz). A partir de este volumen se efectuó la reconstrucción multiplanar (MPR) de la lesión con el software QLAB 10.5 (Philips).

La reconstrucción MPR 3D, confirmó una perforación circular de bordes netos, de dimensiones máximas 6 × 6 $\mathrm{mm}$, localizada en el cuerpo del segmento medio del velo anterior (A2) (Figuras 2 y 3, material complementario disponible en http://www.ecocardio.cl/pmitral.html), sitio desde donde se originaba la insuficiencia descrita. El orificio tenía un ligero comportamiento dinámico durante el ciclo cardíaco, alcanzando una mayor área en telesístole (Figura 4).

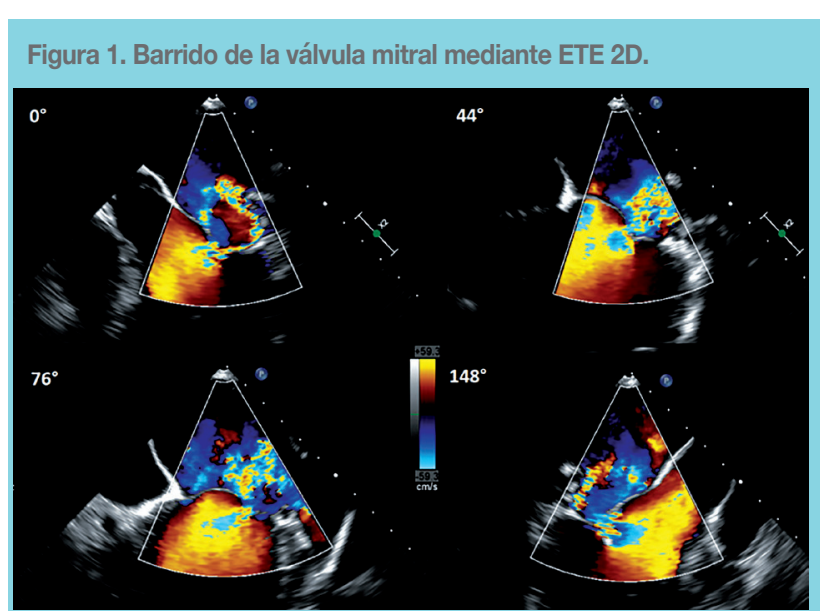

Material complementario disponible en http://www.ecocardio.cl/pmitral.html

Figura 2. Reconstrucción multiplanar de la lesión en A2

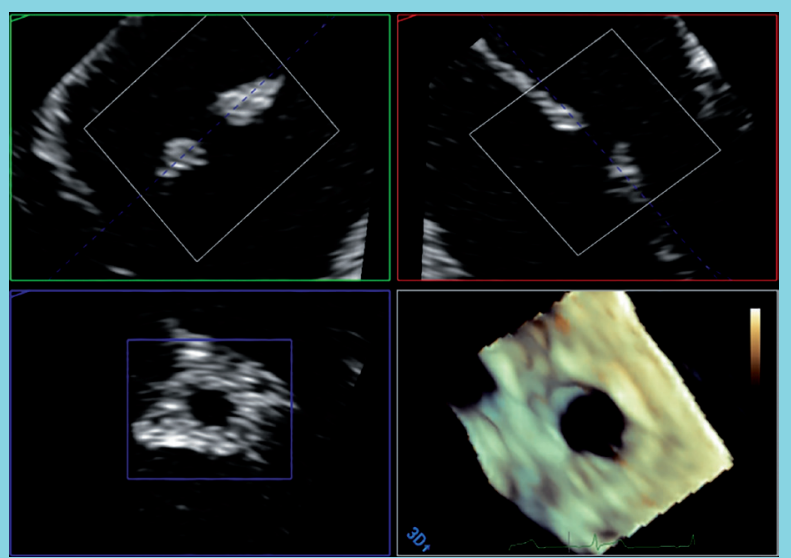

Material complementario disponible en http://www.ecocardio.cl/pmitral.html

Figura 3. Reconstrucción 3D, vista auricular del velo mitral anterior (se ha recortado el velo posterior)

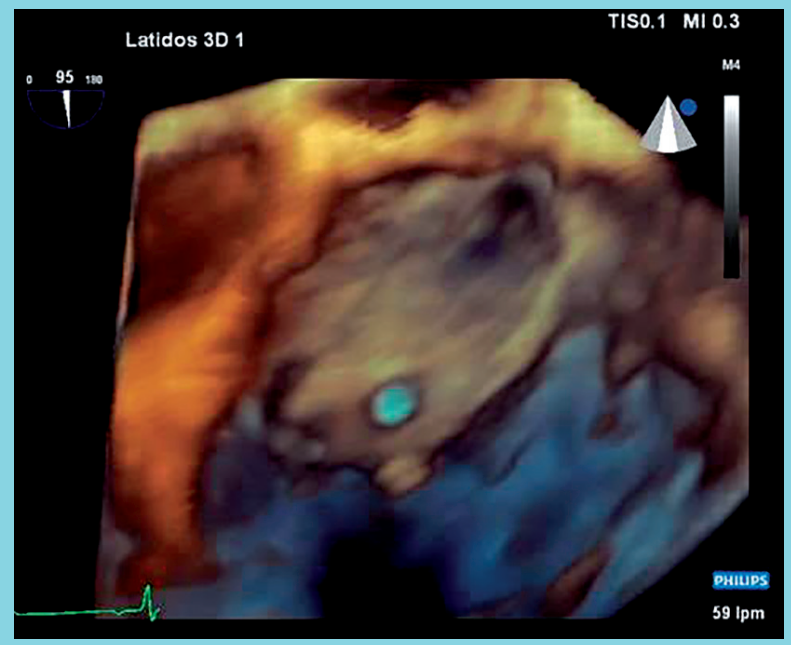

Material complementario disponible en http://www.ecocardio.cl/pmitral.html 
Figura 4, Dinámica de la perforación durante el ciclo cardíaco

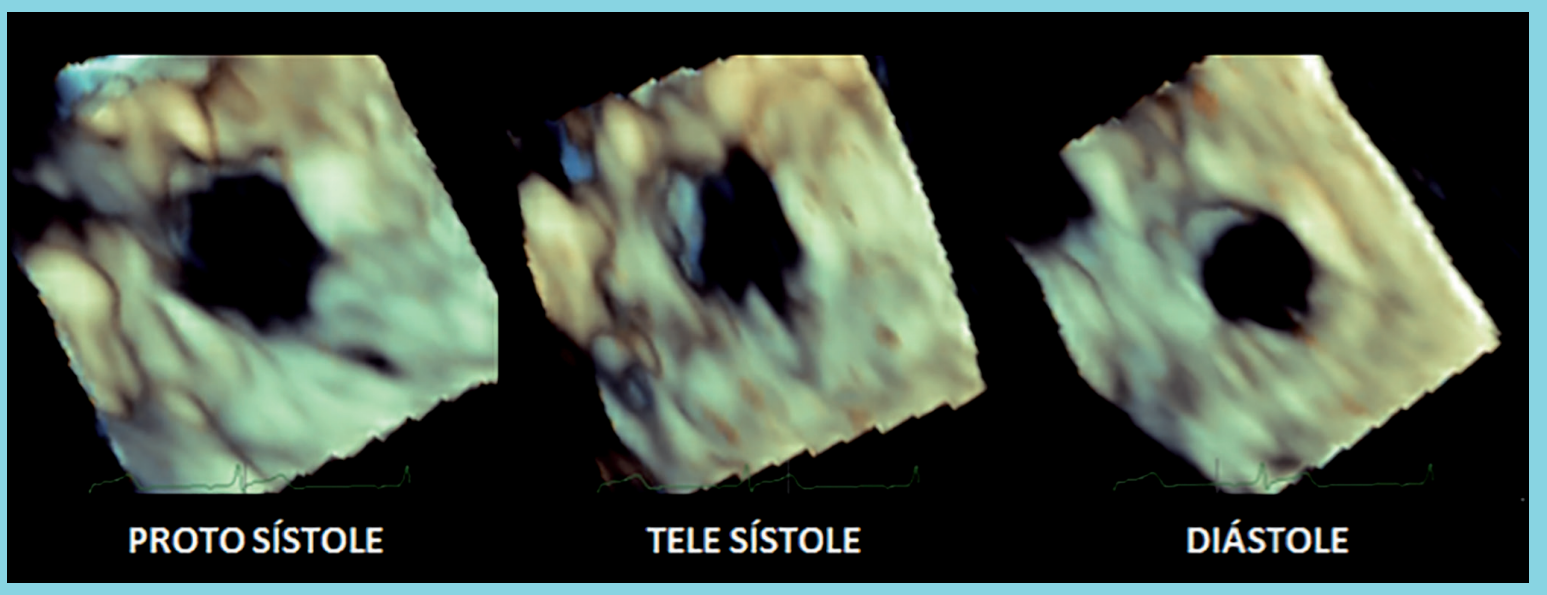

Se decidió tratamiento quirúrgico de la lesión, para lo que se evaluó en conjunto con el equipo de cirugía cardíaca estimándose una alta posibilidad de reparación valvular. Se abordó la válvula mitral mediante un acceso transeptal y superior combinado obteniendo una muy buena exposición. La visualización directa de la lesión coincidió con lo descrito por el ETE 3D. Se visualizó en el cuerpo de A2 una perforación circular de $6 \times 6 \mathrm{~mm}$, con un margen de $3 \mathrm{~mm}$ hacia el borde libre de dicho segmento (Figura 5 panel inferior). Se reparó el defecto con un parche de pericardio autólogo fresco (sin fijación en glutaraldehido) suturado al velo y no a los bordes de la perforación (Figura 5, panel superior). Se complementó la reparación valvular con el implante de un anillo rígido completo de $30 \mathrm{~mm}$ (Carpentier Edwards Physio) (Figura 5 panel superior). El ETE de control a la salida de circulación extracorpórea y el ETT de control no evidenciaron insuficiencia mitral residual, visualizando el parche de pericardio cubriendo el segmento A2. La evolución fue satisfactoria, con alta al 4to día postoperatorio.

\section{Discusión}

El caso que presentamos destaca el rol del ETE 3D en el diagnóstico anatómico preciso de la lesión, permitiendo reconstruir y presentar la válvula mitral al cirujano emulando las condiciones anatómicas de pabellón ("EN FACE"), lo que aporta valiosa información para programar la reparación valvular ${ }^{1}$.

En globo la necesidad de cirugía alejada para tratar las secuelas de una EI oscila entre un $20-40 \%{ }^{2}$. Específicamente, para las lesiones valvulares alejadas, ha sido reportada en un $11 \%$ en una serie de 212 pacientes en un plazo de 15 años ${ }^{3}$, siendo indicada principalmente por
Figura 5. Fotografía de pabellón centrada en la lesión (panel inferior) e ilustración de la reparación (panel superior).

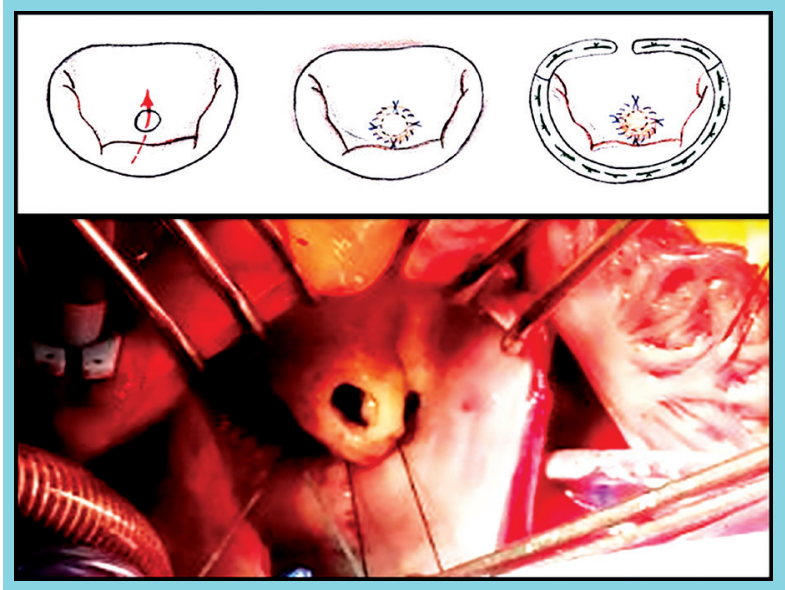

Panel superior: ilustración izquierda: lesión y mecanismo de la insuficiencia. Ilustración central: parche de pericardio fresco suturado al velo. Ilustración derecha: estado final de la reparación con implante de anillo rígido.

insuficiencia aórtica severa y con menor frecuencia para la insuficiencia mitral severa.

La perforación del velo anterior de la válvula mitral ha sido descrita en el contexto de EI como una lesión aguda o como secuela de ésta y puede darse aislada o asociada a una endocarditis aórtica. Hacia los años 90, la EI aguda se consideraba una contraindicación para efectuar una reparación valvular. Sin embargo, tras la serie de Dreyfus y colaboradores ${ }^{4}$ la reparación demostró su excelente resultado y durabilidad, y actualmente se considera como el estándar reparar y no reemplazar la válvula ${ }^{5-6}$, toda vez que ello sea técnicamente posible. La sobrevida libre de eventos con esta técnica es superior a la lograda con el recambio valvular ${ }^{7-8}$. 
En la literatura nacional el 2004 se publicó una experiencia local de 9 años de reparación valvular en $\mathrm{EI}^{9}$, demostrando bajas tasas de morbi-mortalidad y buen resultado alejado de la reparación valvular a un promedio de 3 años de seguimiento. Dicha serie fue actualizada el $2014^{10}$ totalizando 36 reparaciones de válvula mitral en el contexto de una EI. Para ello se utilizaron diversas técnicas quirúrgicas (combinadas o aisladas), que en orden de frecuencia fueron; anuloplastía mitral (89\%), resección cuadrilateral de velo posterior $(33 \%)$, cierre de perforaciones $(22 \%)$, resección de vegetaciones $(22 \%)$ y reparación comisural $(8 \%)$, entre otras. El buen resultado alejado de dichas reparaciones se objetivó por un 92\% libre de insuficiencia significativa a 4.5 años de seguimiento promedio.

Específicamente, la reparación de una perforación del velo anterior con un parche de pericardio ha demostrado excelentes resultados en cuanto a duración y tasa de éxito ${ }^{11}$, con bajos requerimientos de re-operación en el largo plazo ( $<8 \%$ a los 10 años de seguimiento). ${ }^{12}$

Históricamente, el diagnóstico de una perforación valvular mitral mediante Eco 2D TT era un desafío y muchas veces el diagnóstico se realizaba en el intra-ope- ratorio ${ }^{13-14}$. Sin embargo, gracias al desarrollo del ETE 2D y en especial gracias al ETE 3D el rendimiento de la técnica para diagnosticar dichas lesiones se aproximó a un $100 \%$.

El mayor aporte del ETE 3D radica en diagnosticar las insuficiencias mitrales causadas por perforaciones o hendiduras ("cleft") incluidas en el grupo I de Carpentier junto a la dilatación del anillo. En este contexto, el ETE 3-D supera ampliamente al ETE 2D ${ }^{1-4}$. Esto gracias a que el ETE 3-D logra una mejor integración espacial de la anatomía. Sin embargo, su baja resolución temporal sigue siendo una limitación a mejorar, para lo cual es posible utilizar varias técnicas. Por ejemplo; pegando varios latidos y volúmenes para componer una imagen 3D y/o realizando una captura de un sector más pequeño (por ejemplo, capturando solo el velo anterior en su segmento A2). Lo anterior mejora notablemente la resolución temporal manteniendo una óptima resolución espacial, permitiendo representar con fidelidad la situación anatómica de la válvula, incrementando la comprensión de la anatomía valvular por parte del equipo cardioquirúrgico en vista de una eventual reparación, como ocurrió en el caso reportado. 


\section{Referencias}

1. BHAVE NM, ADDETIA K, SPENCER KT, WEINERT L, JEEVANANDAM V, LANG RM. Localizing mitral valve perforations with 3D transesophageal echocardiography. JACC Cardiovasc Imaging. 2013; 6: 407-410.

2. PRENDERGAST BD, TORNOS P. Surgery for infective endocarditis: Who and when? Circulation. 2010; 121: 1141-1152.

3. NETZER ROM, ALTWEGG SC, ZOLLINGER E, TÄUBER M, CARREL T, SEILER C. Infective endocarditis: determinants of long term outcome. Heart. 2002; 88: 61-66.

4. DREYFUS G, SERRAF A, JEBARA VA, DELOCHE A, CHAUVAUD S, COUETIL JP, et al. Valve repair in acute endocarditis. Ann Thorac Surg. 1990; 49: 706-711.

5. BYRNE JG, REZAI K, SANCHEZ JA, BERNSTEIN RA, OKUM E, LEACCHE M, et al. Surgical management of endocarditis: the society of thoracic surgeons clinical practice guideline. Ann Thorac Surg. 2011; 91: 2012-2019.

6. ZHAO D, ZHANG B. Are valve repairs associated with better outcomes than replacements in patients with native active valve endocarditis? Interact Cardiovasc Thorac Surg. 2014;19:10361039.

7. FERINGA HH, SHAW LJ, POLDERMANS D, HOEKS S, VAN DER WALL EE, DION RA, et al. Mitral valve repair and replacement in endocarditis: a systematic review of literature. Ann Thorac Surg. 2007; 83: 564-570.

8. FERINGA HH, BAX JJ, KLEIN P, KLAUTZ RJ, BRAUN J, VAN DER WALL EE, et al. Outcome after mitral valve repair for acute and healed infective endocarditis. Eur J Cardio-thoracic Surg. 2006;29:367-373.
9. ZALAQUETT R, GARRIDO L, CASAS F, MORÁN S, IRARRÁZAVAL M , BECKER P, et al. Cirugía valvular reparadora en endocarditis infecciosa. Rev Med Chil. 2004; 132: 307-315.

10. ZALAQUETT R, LATORRE G, ALMEIDA J, MENESES A, MUÑOZ C, CÓRDOVA S. Veinte años de cirugía reparadora de la válvula mitral. Rev Med Chil. 2014; 142: 1089-1098.

11. EVANS CF, DEFILIPPI CR, SHANG E, GRIFFITH BP, GAMMIE JS. Fresh autologous pericardium for leaflet perforation repair in mitral valve infective endocarditis. J Heart Valve Dis. 2013; 22: 560-566.

12. SHIMOKAWA T, KASEGAWA H, MATSUYAMA S, SEKI H, MANABE S, FUKUI T, et al. Long-term outcome of mitral valve repair for infective endocarditis. Ann Thorac Surg. 2009; 88: 733-739.

13. NOMEIR AM, DOWNES TR, CORDELL AR. Perforation of the anterior mitral leaflet caused by aortic valve endocarditis: diagnosis by two-dimensional, transesophageal echocardiography and color flow Doppler. J Am Soc Echocardiogr. 1992; 5:195-198.

14. MIYATAKE K, YAMAMOTO K, PARK YD, IZUMI S, YAMAGISHI M, SAKAKIBARA H, et al. Diagnosis of mitral valve perforation by real-time two-dimensional Doppler flow imaging technique. J Am Coll Cardiol. 1986; 8: 1235-1239.

15. THOMPSON KA, SHIOTA T, TOLSTRUP K, GURUDEVAN S V, SIEGEL RJ. Utility of three-dimensional transesophageal echocardiography in the diagnosis of valvular perforations. Am J Cardiol. 2011; 107: 100-102. 